\title{
Weber's Law: A Mechanistic Foundation after Two Centuries
}

\section{Other Journal Item}

Author(s):

Brus, Jeroen; Heng, Joseph A.; Polania, Rafael (D)

Publication date:

2019-11

Permanent link:

https://doi.org/10.3929/ethz-b-000376117

\section{Rights / license:}

Creative Commons Attribution-NonCommercial-NoDerivatives 4.0 International

\section{Originally published in:}

Trends in Cognitive Sciences 23(11), https://doi.org/10.1016/j.tics.2019.09.001

\section{Funding acknowledgement:}

758604 - Enhancing brain function and cognition via artificial entrainment of neural oscillations (EC) 


\title{
Weber's law: a mechanistic foundation after two centuries
}

Descriptive words: Decision making, Drift-diffusion model, Psychophysics, Perception

\author{
Authors \\ Jeroen Brus\#, Joseph A. Heng\# \& Rafael Polanía*
}

Decision Neuroscience Lab

Department of Health Sciences and Technology, ETH Zurich, Switzerland

\# Equal contribution

* Corresponding author: Rafael Polanía, rafael.polania@hest.ethz.ch

\section{Acknowledgements}

This work was supported by an ERC starting grant (ENTRAINER) to R.P. This project has received funding from the European Research Council (ERC) under the European Union's Horizon 2020 research and innovation programme (grant agreement No. 758604).

\begin{abstract}
Weber's law appears to be a universal principle describing how we discriminate physical magnitudes. However, this law remained purely descriptive for nearly two centuries. A new study by Pardo-Vazquez et al. finally provides a mechanistic explanation, revealing how both accuracy and reaction time performance lawfully emerge during sensory discrimination tasks.
\end{abstract}

\section{Main text}

Weber's law (WL) [1] is one of the few psychophysical laws that is largely conserved across species and sensory modalities. WL states that when comparing the magnitude of two stimuli, our accuracy does not depend on their absolute difference but rather on the ratio of the compared stimuli. Critically, this law results from empirical observations describing psychophysical performance, while ignoring temporal dynamics underlying the 
discrimination process. Perhaps, this lack of constraint has made it difficult to establish a biologically plausible mechanistic model of WL.

To solve this, Pardo-Vazquez et al. designed an experiment that incorporates the time to make a decision. Specifically, they developed a sound intensity discrimination task performed by rats in which they varied from trial-to-trial the ratios and average magnitude of the two sounds to be discriminated (Figure 1a). They observed that while accuracy is constant for the same intensity ratio at different average magnitudes, the time required to make a decision does not follow this relationship. In particular, input stimuli with higher average magnitudes are more rapidly discriminated (Figure $1 b, c)$, and their reaction time distributions appear to be scale invariant, that is, changes in average magnitude for a given intensity ratio are equivalent to a linear transformation in the units of time used to measure the decision times. Notably, while the importance of reaction times during magnitude discrimination was previously recognized $[3,4]$, the well-controlled experimental setup adopted in this study allowed, for the first time, to provide the tight relationship of scale invariant reaction time distributions and WL. Importantly, the authors rationalized that this apparent strict and joint requirement of accuracies and reaction times could be the hint to establish the mechanistic foundation of WL. To this end, the investigators relied on a general instantiation of a continuous Markov process model allowing to flexibly capture the dynamics of decision making.

First, they investigated the necessary conditions for a continuous Markov process to account for WL's psychophysical performance and the reaction times' scale invariance property. They found only one biologically plausible solution, which required a power-law encoding of stimulus magnitude as well as a linear relationship between the mean and variance of the sensory evidence. Notably, these requirements can be implemented by using populations of neurons with Poisson firing rates. In addition, a fixed decision threshold and the absence of decay in the accumulated evidence were the two remaining necessary conditions. As a result, the model belongs to the class of standard evidence accumulation models, which are commonly used in the decision-making literature [5]. These necessary 
properties not only allow a fundamental understanding of the decision mechanisms, but are also suggested to be implemented by biological systems that instantiate decision processes.

The authors propose a parsimonious implementation of their mechanistic model, which was sufficient to capture the rats' accuracy and reaction time distributions even for data in a range of stimulus intensities that was not used to fit the model parameters. Interestingly, the new theory also generates a counterintuitive prediction about the breakdown of WL. Given that decision evidence evolves slower for lower sound intensities, the theory predicts that early stopping of stimuli presentation should lead to lower accuracies for quieter sounds (Figure $1 \mathrm{~b}, \mathrm{~d}$ ), which clearly violates WL. Strikingly, results of experiments designed to incorporate this manipulation confirmed this counterintuitive prediction.

To provide evidence for the generality of their theory, the researchers showed a similar dependency of accuracies and reaction time distributions in humans on a similar sound discrimination task, and in rodents in an odor-mixture discrimination task. However, whether such a relationship holds for other modalities remains an open question. In particular, higher order percepts (e.g. numerosity discrimination or reward-based decisions), which require integration of information in higher cortical areas, may follow distinct encoding rules from lower order sensory systems such as the ones described in the study. An additional and intriguing result is that rats were unable to adapt the parameters of their decision making process as a function of reward and motivation. They investigated this by changing rewards for correct trials depending on trial difficulty and by presenting only the hardest or easiest trials to the animals. Following principles of optimality, one would expect the animals to adapt their decision thresholds to maximize their reward rate [6]. The authors hypothesize that this lack of adaption could be due to the hardwired nature of neural systems dedicated to detect inter-aural level differences in mammals [7]. Therefore, it could be argued that adaptation to reward distributions in this auditory system may require longer adaptation periods perhaps via top down influences of higher order areas. 
The clear exposition of the mechanisms underlying WL revealed by Pardo-Vazquez et al. generates new questions. For instance, it is unclear how the rigid relationships of accuracies and reaction times found in this study could account for contextual changes in the environment. For the case of the stimuli distributions used by the authors, power-law encoding mechanisms have the convenient property of compressing physical stimulus intensity, allowing neuronal populations with a limited output range to represent a large spectrum of the physical world. As the natural distributions of physical stimuli tend to follow a power-law, such that lower magnitude stimuli are more common than larger ones, power-law encoding allows better discrimination of frequently occurring stimuli, thus efficiently considering the allocation of limited encoding resources. Therefore, power-law encoding of a physical stimulus could be a product of computational principles such as efficient coding, which stems from the limited capacity of neural systems to represent information [8]. This predicts that encoding in sensory systems should adapt if the stimulus distribution changes via experience and learning mechanisms, not only for early sensory perception, but also for higher order processing such as reward systems [9]. However, it is important to note that the sound intensities chosen by Pardo-Vazquez et al. were spaced logarithmically, which may have provided a similar distribution to naturally occurring stimuli. Thus, it would be interesting to place the animals in an environment with a different distribution of sound intensities (e.g., higher intensities occurring more frequently) to test whether WL and reaction time distribution scale invariance still holds.

As we finally move from a purely descriptive to a dynamic mechanistic explanation of $\mathrm{WL}$, an interesting challenge for future research is to understand how these mechanisms can be extended by incorporating learning and adaptation processes. Ultimately, organisms must constantly adapt to dynamic environments for survival. (Un)fortunatly, as WL also applies to time perception [10], the additional decades of research to come will gradually be perceived as shorter and shorter. 
a

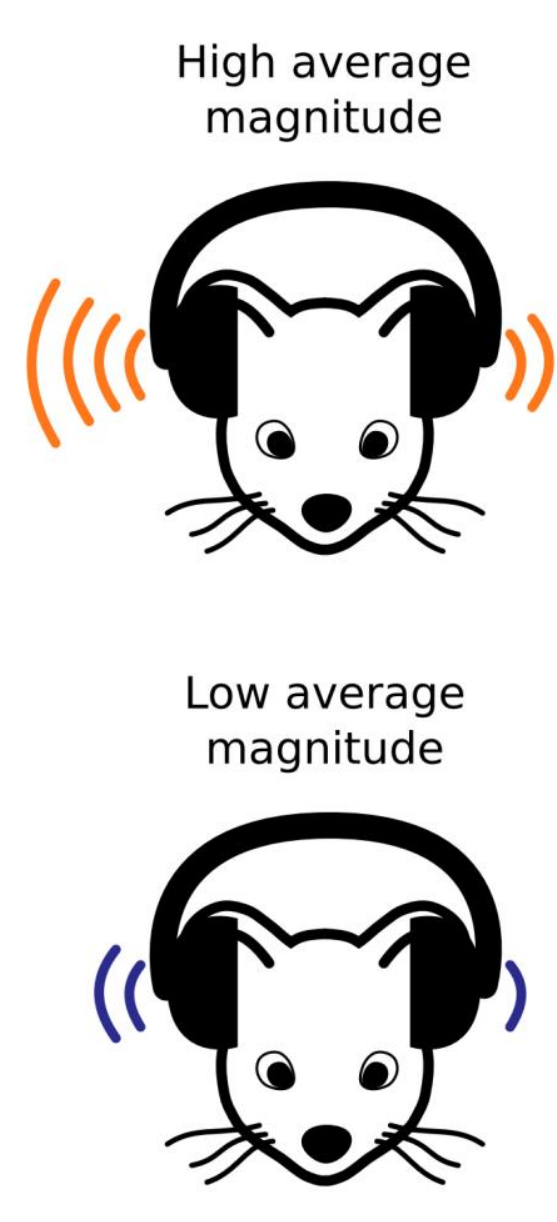

b

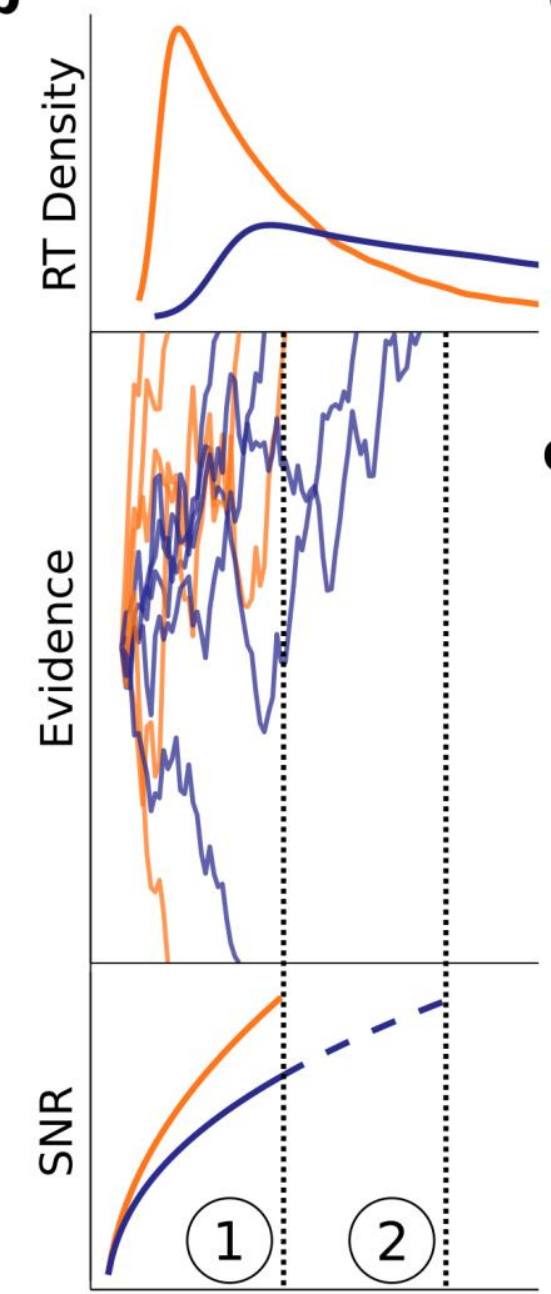

Time
C

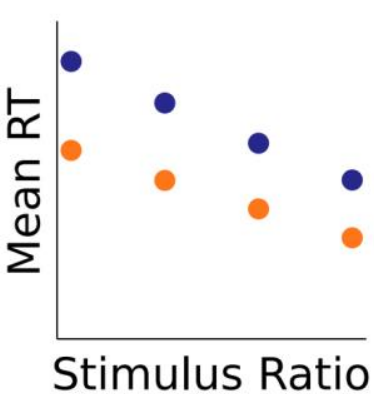

d
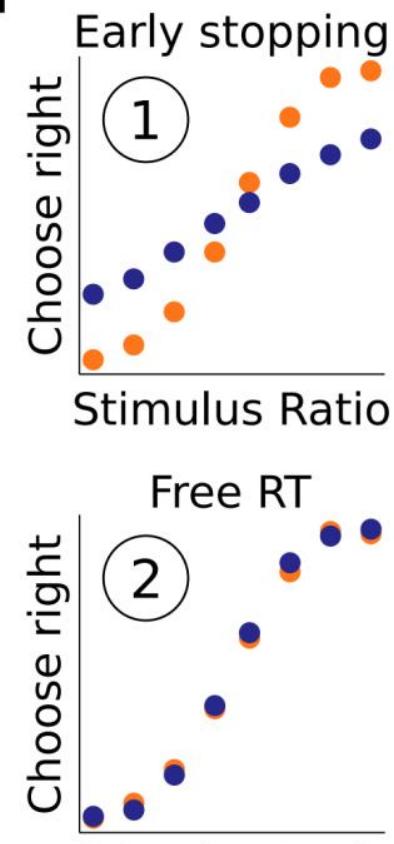

Stimulus Ratio

Figure 1. Dependencies of accuracy and reaction times on stimulus magnitude.

a) Behavioral task: Rats discriminated sounds at various ratios and average magnitudes, high/low magnitudes are in orange/blue. b) Rats accumulate evidence until reaching a decision threshold (middle). Higher magnitudes lead to faster but noisier accumulation, leading to scale invariant reaction time distributions (top). Signal-to-noise ratios at the decision time are identical (bottom). c) Mean reaction times depend on sound ratio and average magnitude. d) If evidence accumulation is stopped early, trials with lower average magnitude will have lower accuracy (1). However, for free reaction times, accuracy follows Weber's law (2). 


\section{References}

1. Weber, E. H. (1834) De Pulsu, Resorptione, Auditu et Tactu: Annotationes Anatomicae et Physiologicae. CF Koehler.

2. Pardo-Vazquez, J.L. et al. (2019) The mechanistic foundation of Weber's law. Nat. Neurosci. 22, 1493-1502

3. Link, S. W. (1992) The Wave Theory of Difference and Similarity. Psychology Press.

4. Simen, P. et al. (2016) Scale (in)variance in a unified diffusion model of decision making and timing. Psychol. Rev. 123, 151-181.

5. Ratcliff, R. et al. (2016) Diffusion Decision Model: Current Issues and History. Trends Cogn. Sci. 20, 260-281

6. Tajima, S. et al. (2016) Optimal policy for value-based decision-making. Nat. Commun. 7, 12400

7. Grothe, B. et al. (2010) Mechanisms of Sound Localization in Mammals. Physiol. Rev. 90, 983-1012

8. Wei, X.-X. and Stocker, A.A. (2017) Lawful relation between perceptual bias and discriminability. Proc. Natl. Acad. Sci. U. S. A. 114, 10244-10249

9. Polanía, R. et al. (2019) Efficient coding of subjective value. Nat. Neurosci. 22, 134142

10. Jazayeri, M. and Shadlen, M.N. (2010) Temporal context calibrates interval timing. Nat. Neurosci. 13, 1020-1026 